\title{
Quantitative inheritance of some wheat plant traits
}

\author{
D. Novoselovic ${ }^{1}$, Marijana Baric ${ }^{2}$, G. Drezner ${ }^{1}$, J. Gunjaca $^{2}$ and A. Lalic ${ }^{1}$ \\ ${ }^{1}$ Agricultural Institute Osijek, Osijek, Croatia. \\ ${ }^{2}$ University of Zagreb, Faculty of Agriculture, Zagreb, Croatia.
}

\begin{abstract}
The objective of this study was to estimate gene effects and genetic variability for some quantitative traits of two winter wheat crosses (Soissons/Zitarka and Soissons/Sana) by generation mean analysis. In most cases a digenic epistatic model was sufficient to explain variation in generation means. The additive-dominance model was adequate for plant height and grain weight per spike of the longest culm. In two cases (grain yield per plant and single grain weight) these models failed to explain variation in generation means, implying the presence of higher order interactions or interactions between linked loci. Dominance effects and additive $\mathrm{x}$ additive epistasis were more important than additive effects and other epistatic components. Only complementary type epistasis was observed. The estimated values of narrow-sense heritability $\left(\mathrm{h}^{2}\right)$ varied for plant height $(54-81 \%)$, number of heads per plant (9-76\%), number of grains per spike (11-99.8\%), grain weight per spike (23-73\%), grain yield per plant (21-78\%) and single grain weight $(49.7-72 \%)$. The adequacy of certain modes of inheritance as well as the importance and significance of gene effects and genetic components of variance for analyzed traits were dependent upon the particular crossing combination and experimental site.
\end{abstract}

Key words: wheat, quantitative traits, joint scaling test, gene effects, heritability.

Received: February 12, 2003; Accepted: August 20, 2003.

\section{Introduction}

Grain yield is a complex trait made up of the interaction between different yield components and environmental effects. Because of these complex interactions it is difficult to improve yield through breeding (especially in the early generations) if yield is the only factor recorded, suggesting that component traits should also be used as selection criteria for yield improvement. This is the reason why it is necessary to know the genetic architecture of yield components (Misra et al., 1994). Generation mean analysis is a simple but useful technique for estimating gene effects for a polygenic trait, its greatest merit lying in the ability to estimate epistatic gene effects such as additive $\mathrm{x}$ additive (aa), dominance $\mathrm{x}$ dominance (dd) and additive $\mathrm{x}$ dominance (ad) effects (Singh and Singh, 1992).

Besides gene effects, breeders would also like to know how much of the variation in a crop is genetic and to what extent this variation is heritable, because efficiency of selection mainly depends on additive genetic variance, influence of the environment and interaction between genotype and environment.

Send correspondence to Dario Novoselovic. Agricultural Institute Osijek, Juzno predgradje 17, Hr-31000 Osijek, Croatia. E-mail: darion@poljinos.hr.
The research reported in this paper was carried out to provide information about gene effects and available genetic variability for the most important quantitative traits of winter wheat (Triticum aestivum L.).

\section{Material and Methods}

We used winter wheat (Triticum aestivum L.), the female parent used in the crosses being cultivar (cv.) Soissons (bred by Desprez, Veuve et Fils, France) and the male parent cv. Sana (bred by the Bc Institute for Plant Breeding and Seed Production, Zagreb, Croatia) and cv. Zitarka (bred by the Agricultural Institute, Osijek, Croatia).

The six basic generations (parent cultivars $\left(\mathrm{P}_{1}, \mathrm{P}_{2}\right)$, first and second filial generations $\left(\mathrm{F}_{1}, \mathrm{~F}_{2}\right)$, first and second backcrosses $\left.\left(\mathrm{BC}_{1}, \mathrm{BC}_{2}\right)\right)$ of two combinations of crossings (Soissons/Zitarka and Soissons/Sana) were planted in a randomized block design in two replicate plots at two sites (Osijek and Nova Gradiska, Croatia) during the autumn season of 1999/2000. The experimental field layout was rectangular at the Osijek site and square at the Nova Gradiska site. The plots were $1 \mathrm{~m}$ long with a between-row spacing of $20 \mathrm{~cm}$ and a within-row spacing of $10 \mathrm{~cm}$. The number of rows per plot and the number of analyzed plants per plot varied depending on the generation. 
The traits assessed were: plant height $(\mathrm{cm})$; number of heads per plant; number of grains per spike of the longest culm; grain weight per spike of the longest culm (g); grain yield per plant $(\mathrm{g})$ and single grain weight $(\mathrm{mg})$, i.e. grain weight per spike of the longest culm divided by the number of grains on that particular spike.

The mean values, standard errors and variances of the different generations were subjected to weighted least squares analysis using the joint scaling test (Mather and Jinks, 1982) to estimate gene effects and the maximum likelihood method (Hayman, 1960) to estimate variance components to fit models of increasing complexity until an adequate description of the observed means were found as shown by non-significance in the $\chi^{2}$ test. The significance of genetic parameters was tested by t-test.

The type of epistasis was determined only when dominance (d) and dominance $\mathrm{x}$ dominance (dd) effects were significant, when these effects had the same sign the effects were complementary while different signs indicated duplicate epistasis (Kearsey and Pooni, 1996).

Homogeneity of variances of non-segregating generations was tested for using Bartlett's test (Bartlett, 1937), and when the variances were heterogeneous the environmental variance $\left(\mathrm{V}_{\mathrm{E}}\right)$ was replaced by an adequate number of separate parameters in the model fitting and pooled to produce a single environmental variance. Additive, dominance and environmental variance components were estimated using the maximum likelihood method with the observed variances of the six basic generations being used as the initial weights $\left(\mathrm{df} / 2 * \mathrm{~s}^{2}+2\right)$ until the $\chi^{2}$ test values reached a minimum (Lynch and Walsh, 1998).

Narrow-sense heritability $\left(\mathrm{h}_{\mathrm{n}}^{2}\right)$ was calculated as follows:

$\mathrm{h}_{\mathrm{n}}^{2}=\mathrm{V}^{*}{ }_{\mathrm{A}} / \mathrm{V}^{*}{ }_{\mathrm{A}}+\mathrm{V}^{*}{ }_{\mathrm{D}}+\mathrm{V}_{\mathrm{E}}$, where $\mathrm{V}^{*}{ }_{\mathrm{A}}=0.5 *^{*} \mathrm{a}_{\mathrm{A}}$ is the additive genetic component of variance, $\mathrm{V}^{*}{ }_{\mathrm{D}}=$ $0.25 * \mathrm{~d}_{\mathrm{A}}^{2}$ the dominance or non-additive genetic component of variance and $V_{E}$ the environmental variance (Kearsey and Pooni, 1996). The $\mathrm{V}^{*}{ }_{\mathrm{D}}$ value was set to zero when estimated variance turned out to be a negative.

All statistical analyses were carried out using the STAT module and PROC REG procedure of the SAS software (SAS Institute, 1996).

\section{Results}

The mean values, their standard errors and variances for the analyzed traits of the two crosses at the two sites are presented in Tables 1 and 2. The parents of the Soissons/ Zitarka cross differed in respect to the number of heads per plant, grain yield per plant and single grain weight at both sites, although for the $F_{1}$ generation only the means for the number of grains and grain weight per spike of the longest culm and single grain weight were greater than the better parent, and this occurred only at the Nova Gradiska site. For the other traits, differences were not so conspicuous
Table 1 - Quantitative trait data for the Soissons/Zitarka cross at the Osijek and Nova Gradiska sites.

\begin{tabular}{|c|c|c|c|c|}
\hline \multirow[b]{3}{*}{ Generation $^{1}$} & \multicolumn{4}{|c|}{ Quantitative trait data for the Soissons/Zitarka cross } \\
\hline & \multicolumn{2}{|c|}{ Osijek site } & \multicolumn{2}{|c|}{ Nova Gradiska site } \\
\hline & Mean $\pm \mathrm{SE}^{2}$ & Variance & Mean \pm SE & Varianc \\
\hline \multicolumn{5}{|c|}{ Plant height $(\mathrm{cm})$} \\
\hline $\mathrm{P}_{1}$ & $64.3 \pm 0.69$ & 30.47 & $75.3 \pm 0.29$ & 5.80 \\
\hline $\mathrm{P}_{2}$ & $65.0 \pm 0.58$ & 22.88 & $68.4 \pm 0.36$ & 8.68 \\
\hline $\mathrm{F}_{1}$ & $63.9 \pm 0.78$ & 42.59 & $70.7 \pm 0.54$ & 28.58 \\
\hline $\mathrm{F}_{2}$ & $70.0 \pm 0.44$ & 75.31 & $73.6 \pm 0.38$ & 48.95 \\
\hline $\mathrm{BC}_{1}$ & $64.0 \pm 0.88$ & 49.28 & $72.1 \pm 0.52$ & 23.79 \\
\hline $\mathrm{BC}_{2}$ & $67.1 \pm 0.60$ & 31.32 & $71.6 \pm 0.50$ & 23.25 \\
\hline \multicolumn{5}{|c|}{ Number of heads per plant } \\
\hline $\mathrm{P}_{1}$ & $11.0 \pm 0.34$ & 7.39 & $9.0 \pm 0.25$ & 4.31 \\
\hline $\mathrm{P}_{2}$ & $7.6 \pm 0.23$ & 3.59 & $6.9 \pm 0.21$ & 2.95 \\
\hline $\mathrm{F}_{1}$ & $8.6 \pm 0.35$ & 8.58 & $6.8 \pm 0.23$ & 5.18 \\
\hline $\mathrm{F}_{2}$ & $10.8 \pm 0.21$ & 17.15 & $7.0 \pm 0.11$ & 4.10 \\
\hline $\mathrm{BC}_{1}$ & $9.0 \pm 0.28$ & 6.04 & $7.6 \pm 0.19$ & 3.18 \\
\hline $\mathrm{BC}_{2}$ & $8.5 \pm 0.24$ & 5.01 & $7.9 \pm 0.24$ & 5.36 \\
\hline
\end{tabular}

Number of grains per spike of the longest culm

$\begin{array}{lrrrr}\mathrm{P}_{1} & 58.5 \pm 1.23 & 96.83 & 53.8 \pm 0.89 & 54.65 \\ \mathrm{P}_{2} & 58.3 \pm 0.92 & 57.56 & 52.1 \pm 0.91 & 55.48 \\ \mathrm{~F}_{1} & 55.4 \pm 1.09 & 83.17 & 55.9 \pm 1.05 & 108.05 \\ \mathrm{~F}_{2} & 58.7 \pm 0.70 & 190.61 & 52.5 \pm 0.59 & 118.00 \\ \mathrm{BC}_{1} & 61.1 \pm 1.31 & 132.14 & 53.1 \pm 1.12 & 110.39 \\ \mathrm{BC}_{2} & 60.4 \pm 0.93 & 75.25 & 53.6 \pm 0.96 & 85.71\end{array}$

Grain weight per spike of the longest culm (g)

\begin{tabular}{lrrrr}
$\mathrm{P}_{1}$ & $2.55 \pm 0.06$ & 36.97 & $2.18 \pm 0.04$ & 0.11 \\
$\mathrm{P}_{2}$ & $2.72 \pm 0.05$ & 6.54 & $2.20 \pm 0.04$ & 0.11 \\
$\mathrm{~F}_{1}$ & $2.47 \pm 0.06$ & 24.37 & $2.42 \pm 0.05$ & 0.25 \\
$\mathrm{~F}_{2}$ & $2.61 \pm 0.04$ & 130.86 & $2.26 \pm 0.03$ & 0.31 \\
$\mathrm{BC}_{1}$ & $2.61 \pm 0.06$ & 43.31 & $2.23 \pm 0.05$ & 0.22 \\
$\mathrm{BC}_{2}$ & $2.80 \pm 0.05$ & 29.27 & $2.22 \pm 0.04$ & 0.15 \\
$\mathrm{Grain}$ yield per plant $(\mathrm{g})$ & & & \\
$\mathrm{P}_{1}$ & $20.37 \pm 0.83$ & 44.09 & $17.88 \pm 0.60$ & 24.84 \\
$\mathrm{P}_{2}$ & $14.36 \pm 0.53$ & 19.10 & $10.97 \pm 0.39$ & 10.19 \\
$\mathrm{~F}_{1}$ & $16.76 \pm 0.83$ & 48.22 & $13.57 \pm 0.54$ & 28.58 \\
$\mathrm{~F}_{2}$ & $20.96 \pm 0.52$ & 105.19 & $13.42 \pm 0.26$ & 22.92 \\
$\mathrm{BC}_{1}$ & $15.89 \pm 0.66$ & 33.54 & $14.37 \pm 0.51$ & 22.89 \\
$\mathrm{BC}_{2}$ & $17.68 \pm 0.63$ & 34.53 & $13.75 \pm 0.44$ & 18.00 \\
$\mathrm{Single}_{2}$ grain & weight (mg) & & & \\
$\mathrm{P}_{1}$ & $43.55 \pm 0.49$ & 15.37 & $40.56 \pm 0.35$ & 8.45 \\
$\mathrm{P}_{2}$ & $46.63 \pm 0.43$ & 12.57 & $42.23 \pm 0.34$ & 7.75 \\
$\mathrm{~F}_{1}$ & $44.51 \pm 0.52$ & 18.93 & $43.25 \pm 0.33$ & 10.67 \\
$\mathrm{~F}_{2}$ & $44.16 \pm 0.30$ & 35.01 & $43.12 \pm 0.23$ & 17.93 \\
$\mathrm{BC}_{1}$ & $42.68 \pm 0.46$ & 16.29 & $42.15 \pm 0.41$ & 14.79 \\
$\mathrm{BC}_{2}$ & $46.26 \pm 0.40$ & 13.92 & $41.53 \pm 0.38$ & 13.43 \\
\hline
\end{tabular}

${ }^{1} \mathrm{P}_{1}, \mathrm{P}_{2}=$ parent cultivars; $\mathrm{F}_{1}, \mathrm{~F}_{2}=$ first and second filial generations; $\mathrm{BC}_{1}$, $\mathrm{BC}_{2}=$ first and second backcrosses.

${ }^{2} \mathrm{SE}=$ standard error. 
Table 2 - Quantitative trait data for the Soissons/Sana cross at the Osijek and Nova Gradiska sites.

\begin{tabular}{|c|c|c|c|c|}
\hline \multirow[b]{3}{*}{ Generation $^{1}$} & \multicolumn{4}{|c|}{ Quantitative trait data for the Soissons/Sana cross } \\
\hline & \multicolumn{2}{|c|}{ Osijek site } & \multicolumn{2}{|c|}{ Nova Gradiska site } \\
\hline & Mean $\pm \mathrm{SE}^{2}$ & Variance & Mean $\pm \mathrm{SE}$ & Variance \\
\hline \multicolumn{5}{|c|}{ Plant height (cm) } \\
\hline $\mathrm{P}_{1}$ & $73.1 \pm 0.55$ & 22.69 & $73.8 \pm 0.33$ & 8.28 \\
\hline $\mathrm{P}_{2}$ & $67.0 \pm 0.54$ & 15.45 & $65.1 \pm 0.50$ & 15.50 \\
\hline $\mathrm{F}_{1}$ & $73.0 \pm 1.13$ & 79.17 & $75.3 \pm 0.60$ & 28.80 \\
\hline $\mathrm{F}_{2}$ & $78.1 \pm 0.41$ & 57.99 & $73.3 \pm 0.45$ & 66.62 \\
\hline $\mathrm{BC}_{1}$ & $76.0 \pm 0.46$ & 19.04 & $75.1 \pm 0.46$ & 13.75 \\
\hline $\mathrm{BC}_{2}$ & $70.9 \pm 0.62$ & 30.37 & $71.2 \pm 0.61$ & 29.39 \\
\hline \multicolumn{5}{|c|}{ Number of heads per plant } \\
\hline $\mathrm{P}_{1}$ & $9.5 \pm 0.27$ & 5.47 & $7.9 \pm 0.24$ & 4.38 \\
\hline $\mathrm{P}_{2}$ & $8.4 \pm 0.27$ & 3.86 & $7.5 \pm 0.26$ & 4.19 \\
\hline $\mathrm{F}_{1}$ & $10.4 \pm 0.46$ & 13.12 & $7.9 \pm 0.21$ & 3.53 \\
\hline $\mathrm{F}_{2}$ & $8.8 \pm 0.14$ & 6.76 & $7.4 \pm 0.15$ & 7.40 \\
\hline $\mathrm{BC}_{1}$ & $9.4 \pm 0.23$ & 4.76 & $7.9 \pm 0.26$ & 4.39 \\
\hline $\mathrm{BC}_{2}$ & $8.3 \pm 0.25$ & 4.94 & $8.1 \pm 0.23$ & 4.18 \\
\hline \multicolumn{5}{|c|}{ Number of grains per spike of the longest culm } \\
\hline $\mathrm{P}_{1}$ & $58.8 \pm 0.98$ & 72.03 & $55.8 \pm 1.11$ & 93.64 \\
\hline $\mathrm{P}_{2}$ & $61.5 \pm 1.37$ & 99.48 & $58.1 \pm 1.51$ & 141.37 \\
\hline $\mathrm{F}_{1}$ & $60.9 \pm 1.30$ & 104.78 & $55.7 \pm 1.05$ & 88.20 \\
\hline $\mathrm{F}_{2}$ & $54.2 \pm 0.75$ & 194.06 & $50.8 \pm 0.76$ & 190.03 \\
\hline $\mathrm{BC}_{1}$ & $58.5 \pm 0.95$ & 81.23 & $54.7 \pm 1.09$ & 77.23 \\
\hline $\mathrm{BC}_{2}$ & $61.0 \pm 1.15$ & 104.48 & $57.8 \pm 1.34$ & 141.85 \\
\hline \multicolumn{5}{|c|}{ Grain weight per spike of the longest culm (g) } \\
\hline $\mathrm{P}_{1}$ & $2.48 \pm 0.05$ & 0.19 & $2.29 \pm 0.05$ & 0.19 \\
\hline $\mathrm{P}_{2}$ & $2.88 \pm 0.08$ & 0.34 & $2.18 \pm 0.06$ & 0.22 \\
\hline $\mathrm{F}_{1}$ & $2.88 \pm 0.09$ & 0.50 & $2.40 \pm 0.04$ & 0.13 \\
\hline $\mathrm{F}_{2}$ & $2.38 \pm 0.04$ & 0.55 & $2.14 \pm 0.04$ & 0.53 \\
\hline $\mathrm{BC}_{1}$ & $2.58 \pm 0.05$ & 0.23 & $2.45 \pm 0.05$ & 0.16 \\
\hline $\mathrm{BC}_{2}$ & $2.64 \pm 0.06$ & 0.28 & $2.35 \pm 0.05$ & 0.20 \\
\hline \multicolumn{5}{|c|}{ Grain yield per plant (g) } \\
\hline $\mathrm{P}_{1}$ & $16.92 \pm 0.62$ & 28.83 & $15.24 \pm 0.58$ & 25.57 \\
\hline $\mathrm{P}_{2}$ & $19.19 \pm 0.77$ & 31.42 & $12.99 \pm 0.48$ & 14.28 \\
\hline $\mathrm{F}_{1}$ & $23.01 \pm 1.44$ & 128.56 & $16.53 \pm 0.57$ & 25.99 \\
\hline $\mathrm{F}_{2}$ & $16.90 \pm 0.38$ & 49.82 & $13.78 \pm 0.32$ & 33.69 \\
\hline $\mathrm{BC}_{1}$ & $18.62 \pm 0.57$ & 29.24 & $15.42 \pm 0.60$ & 23.40 \\
\hline $\mathrm{BC}_{2}$ & $17.66 \pm 0.60$ & 28.44 & $15.49 \pm 0.52$ & 21.36 \\
\hline \multicolumn{5}{|c|}{ Single grain weight (mg) } \\
\hline $\mathrm{P}_{1}$ & $42.09 \pm 0.38$ & 10.83 & $41.14 \pm 0.29$ & 6.39 \\
\hline $\mathrm{P}_{2}$ & $46.69 \pm 0.45$ & 10.73 & $37.60 \pm 0.43$ & 11.46 \\
\hline $\mathrm{F}_{1}$ & $47.04 \pm 0.72$ & 32.14 & $43.14 \pm 0.30$ & 7.20 \\
\hline $\mathrm{F}_{2}$ & $43.63 \pm 0.33$ & 37.57 & $42.0 \pm 0.28$ & 25.79 \\
\hline $\mathrm{BC}_{1}$ & $43.96 \pm 0.36$ & 11.66 & $44.91 \pm 0.44$ & 12.58 \\
\hline $\mathrm{BC}_{2}$ & $43.38 \pm 0.58$ & 26.58 & $40.99 \pm 0.46$ & 16.72 \\
\hline
\end{tabular}

${ }^{1} \mathrm{P}_{1}, \mathrm{P}_{2}=$ parent cultivars; $\mathrm{F}_{1}, \mathrm{~F}_{2}=$ first and second filial generations; $\mathrm{BC}_{1}$, $\mathrm{BC}_{2}=$ first and second backcrosses.

${ }^{2} \mathrm{SE}=$ standard error. and consistent. The observed variances were lower at the Nova Gradiska site for all analyzed traits (Table 1). For the Soissons/Sana cross, at both sites, parents differed in respect of plant height and number of grains per spike of the longest culm but, depending on the site, differences between these parents were inconsistent for grain weight per spike of the longest culm, grain yield per plant and single grain weight. For the number of heads per plant differences were not so conspicuous at either site. At both sites the mean grain yield per plant and single grain weight of the $F_{1}$ generation of this cross was greater than that of the better parent, but the means for plant height and grain weight per spike of the longest culm of the $F_{1}$ generation were greater than the corresponding means for the parents only at the Nova Gradiska site, while the mean number of heads per plant was greater for the $F_{1}$ generation only at the Osijek site. The variances were lower at the Nova Gradiska site for all the analyzed traits except number of grains per spike of the longest culm where the differences were not so consistent (Table 2).

The estimates of the main and first order interaction gene effects and $\chi^{2}$ values are presented in Table 3 .

For plant height, an additive-dominance model was adequate only for the Soissons/Sana cross at the Nova Gradiska site, while a digenic epistatic model was adequate for the Soissons/Sana cross at the Osijek site and the Soissons/Zitarka cross at both sites (non-significant $\chi^{2}$ test), with dominance effects and additive $\mathrm{x}$ additive epistasis being more important than other genetic effects and complementary epistasis being found only in the Soissons/Sana cross at the Osijek site. For the number of heads per plant and the number of grains per spike of the longest culm the digenic epistatic model was adequate for both crosses and for both sites, with dominance effects and dominance $\mathrm{x}$ dominance epistasis, when present, being more important as compared to other genetic effects. Except for the Soissons/Sana cross at the Osijek site, both crosses showed complementary epistasis for the number of heads per plant. For the number of grains per spike of the longest culm complementary epistasis was only found in the cross Soissons/Sana at the Osijek site. For grain weight per spike of the longest culm, an additive-dominance model adequately explained variation between generations of the Soissons/Zitarka cross, while a digenic epistatic model was applicable to the Soissons/Sana cross at both sites, although complementary epistasis was found only in the Soissons/ Sana cross at the Nova Gradiska site. For grain yield per plant, both models failed to explain variation between generations (significant $\chi^{2}$ test) for the Soissons/Zitarka cross at the Osijek site, with all three epistasis components being significant and both negative and complementary epistasis also being found. However, at the Nova Gradiska site a digenic epistatic model adequately explained variation between the Soissons/Zitarka cross generations regarding 
grain yield per plant, although only additive effects were significant.

For the Soissons/Sana cross, grain yield per plant was adequately explained by a digenic epistatic model with dominance effects and additive $\mathrm{x}$ additive epistasis being most important. For single grain weight, the digenic epistatic model was satisfactory for the Soissons/Zitarka and Soissons/Sana crosses at the Osijek site, although both the additive-dominance model and the digenic epistatic model failed to explain variation in single grain weight in the Soissons/Sana cross at the Nova Gradiska site where all three epistasis components were significant and positive. Complementary type of epistasis was only found in the Soissons/Zitarka cross at the Nova Gradiska site (Table 3).

The estimates of the different variance components and narrow-sense heritability $\left(\mathrm{h}_{\mathrm{n}}^{2}\right)$ are presented in Table 4 . Maximum likelihood estimates of environmental variance were higher at the Osijek site than the Nova Gradiska site for all traits and crosses except the number of grains per spike of the longest culm for the Soissons/Zitarka cross.

Table 3 - The estimates of gene effects for six quantitative traits in two crossing combinations and sites.

\begin{tabular}{|c|c|c|c|c|c|c|c|c|c|c|}
\hline Trait & Cross & Site & $\mathrm{m}$ & a & d & aa & $\mathrm{dd}$ & ad & $\underset{(\mathrm{df})^{1}}{\chi^{2}}$ & $\begin{array}{l}\text { Type of } \\
\text { epistasis }\end{array}$ \\
\hline \multirow[t]{4}{*}{ Plant height $(\mathrm{cm})$} & \multirow[t]{2}{*}{$\begin{array}{l}\text { Soissons/ } \\
\text { Zitarka }\end{array}$} & Osijek & $76.0^{* * *}$ & 0.39 & $\begin{array}{l}-12.7 * * \\
*\end{array}$ & $-11.5^{* * *}$ & -5.3 & - & $3.76(1)$ & - \\
\hline & & Nova Gradiska & $78.7 * * *$ & 3.2 & $-8.0^{* * *}$ & $-6.8 * * *$ & -4.2 & - & $3.64(1)$ & - \\
\hline & \multirow[t]{2}{*}{$\begin{array}{l}\text { Soissons/ } \\
\text { Sana }\end{array}$} & Osijek & $87.5^{* * *}$ & 3.5 & $\begin{array}{l}-14.5^{* *} \\
*\end{array}$ & $-17.5^{* * *}$ & $-8.8^{*}$ & - & $1.72(1)$ & $\begin{array}{l}\text { Comple- } \\
\text { mentary }\end{array}$ \\
\hline & & Nova Gradiska & $69.7 * * *$ & 4.3 & $6.4 * * *$ & - & - & - & $3.59(3)$ & - \\
\hline \multirow[t]{4}{*}{$\begin{array}{l}\text { Number of heads per } \\
\text { plant }\end{array}$} & \multirow[t]{2}{*}{$\begin{array}{l}\text { Soissons/ } \\
\text { Zitarka }\end{array}$} & Osijek & $17.2 * * *$ & $1.43^{* * *}$ & $-8.6 * * *$ & $-8.0 * * *$ & $-8.4 * * *$ & - & $0.63(1)$ & $\begin{array}{l}\text { Comple- } \\
\text { mentary }\end{array}$ \\
\hline & & Nova Gradiska & $5.0 * * *$ & $0.73 * * *$ & $1.8^{*}$ & $2.9 * * *$ & $4.3^{* *}$ & - & $0.77(1)$ & $\begin{array}{l}\text { Comple- } \\
\text { mentary }\end{array}$ \\
\hline & \multirow{2}{*}{$\begin{array}{l}\text { Soissons/ } \\
\text { Sana }\end{array}$} & Osijek & $7.5 * * *$ & $0.65 * * *$ & $2.5 * *$ & $1.4^{*}$ & - & - & $5.33(2)$ & - \\
\hline & & Nova Gradiska & $5.0^{* * *}$ & 0.13 & $2.87^{* *}$ & $2.67^{* *}$ & $3.62 *$ & - & $0.06(1)$ & $\begin{array}{l}\text { Comple- } \\
\text { mentary }\end{array}$ \\
\hline \multirow[t]{4}{*}{$\begin{array}{l}\text { Number of grains per } \\
\text { spike of the longest culm }\end{array}$} & \multirow[t]{2}{*}{$\begin{array}{l}\text { Soissons/ } \\
\text { Zitarka }\end{array}$} & Osijek & $50.6^{* * *}$ & 0.19 & 4.8 & 7.8 & $22.5^{* *}$ & - & $0.17(1)$ & - \\
\hline & & Nova Gradiska & $49.1 * * *$ & 0.62 & $6.75^{* * *}$ & $3.81^{*}$ & - & - & $0.67(2)$ & - \\
\hline & \multirow[t]{2}{*}{$\begin{array}{l}\text { Soissons/ } \\
\text { Sana }\end{array}$} & Osijek & $47.9^{* * *}$ & $1.6^{*}$ & $14.2 * * *$ & $12.8 * * *$ & $10.9^{* * *}$ & - & $6.98(2)$ & $\begin{array}{l}\text { Comple- } \\
\text { mentary }\end{array}$ \\
\hline & & Nova Gradiska & $46.7 * * *$ & 1.6 & $9.7 * * *$ & $10.9^{* * *}$ & - & - & $7.13(2)$ & - \\
\hline \multirow[t]{4}{*}{$\begin{array}{l}\text { Grain weight per spike of } \\
\text { the longest culm }\end{array}$} & \multirow[t]{2}{*}{$\begin{array}{l}\text { Soissons/ } \\
\text { Zitarka }\end{array}$} & Osijek & $2.7 * * *$ & $0.11 * *$ & -0.11 & - & - & - & $9.9(3)$ & - \\
\hline & & Nova Gradiska & $2.2 * * *$ & 0.01 & $0.19^{* * *}$ & - & - & - & $3.72(3)$ & - \\
\hline & \multirow{2}{*}{$\begin{array}{l}\text { Soissons/ } \\
\text { Sana }\end{array}$} & Osijek & $1.9^{* * *}$ & $0.17 * * *$ & $1.04 * * *$ & $0.81^{* * *}$ & - & - & $3.16(2)$ & - \\
\hline & & Nova Gradiska & $1.2 * * *$ & $0.07^{*}$ & $1.2 * * *$ & $1.1^{* * *}$ & $1.4^{* * *}$ & - & $0.001(1)$ & $\begin{array}{l}\text { Comple- } \\
\text { mentary }\end{array}$ \\
\hline \multirow[t]{4}{*}{ Grain yield per plant (g) } & \multirow[t]{2}{*}{$\begin{array}{l}\text { Soissons/ } \\
\text { Zitarka }\end{array}$} & Osijek & $34.1 * * *$ & $3.0^{* * *}$ & $-7.7^{*}$ & $-16.7 * * *$ & $\begin{array}{l}-37.0^{* *} \\
*\end{array}$ & $-9.6 * * *$ & $(0) * *$ & $\begin{array}{l}\text { Comple- } \\
\text { mentary }\end{array}$ \\
\hline & & Nova Gradiska & $11.0 * * *$ & $2.8^{* * *}$ & 2.6 & 3.2 & 4.6 & - & $3.39(1)$ & - \\
\hline & \multirow{2}{*}{$\begin{array}{l}\text { Soissons/ } \\
\text { Sana }\end{array}$} & Osijek & $11.3^{* * *}$ & 0.58 & $10.9 * * *$ & $6.5^{* * *}$ & - & - & $5.44(2)$ & - \\
\hline & & Nova Gradiska & $11.2^{* * *}$ & $0.89^{* *}$ & $5.8^{* * *}$ & $3.1^{* *}$ & - & - & $6.33(2)$ & - \\
\hline \multirow[t]{4}{*}{ Single grain weight (mg) } & \multirow{2}{*}{$\begin{array}{l}\text { Soissons/ } \\
\text { Zitarka }\end{array}$} & Osijek & $43.8^{* * *}$ & $1.99 * * *$ & 0.74 & 1.2 & - & - & $8.71(2)$ & - \\
\hline & & Nova Gradiska & $46.8^{* * *}$ & $0.6^{* *}$ & $-3.5^{*}$ & $-5.4 * * *$ & $-7.6^{* *}$ & - & $0.86(1)$ & $\begin{array}{l}\text { Comple- } \\
\text { mentary }\end{array}$ \\
\hline & \multirow{2}{*}{$\begin{array}{l}\text { Soissons/ } \\
\text { Sana }\end{array}$} & Osijek & $42.0^{* * *}$ & $1.8^{* * *}$ & $5.0^{*}$ & 2.3 & -3.6 & - & $3.97(1)$ & - \\
\hline & & Nova Gradiska & $35.6^{* * *}$ & $1.8^{* *}$ & 3.3 & $3.8^{*}$ & $19.2^{* *}$ & $4.3^{*}$ & $(0) * *$ & - \\
\hline
\end{tabular}

${ }^{1} \mathrm{df}=$ degrees of freedom, calculated as the number of generations minus the number of estimated genetic parameters.

$*=$ statistically significant at $\mathrm{p}=0.95$.

$* *=$ statistically significant at $\mathrm{p}=0.99$.

$* * *=$ statistically significant at $\mathrm{p}=0.999$. 
Table 4 - Estimation of variance components and narrow-sense heritability $\left(\mathrm{h}^{2}\right)$ for some quantitative traits of the Soissons/Zitarka and Soissons/Sana crosses at the Osijek and Nova Gradiska sites.

\begin{tabular}{|c|c|c|c|c|}
\hline \multirow{3}{*}{ Variance components } & \multicolumn{4}{|c|}{ Quantitative trait data } \\
\hline & \multicolumn{2}{|l|}{ Soissons/Zitarka cross } & \multicolumn{2}{|c|}{ Soissons/Sana cross } \\
\hline & Osijek site & Nova Gradiska site & Osijek site & Nova Gradiska site \\
\hline \multicolumn{5}{|l|}{ Plant height $(\mathrm{cm})$} \\
\hline Phenotypic variance & 93.67 & 66.09 & 107.83 & 108.5 \\
\hline Environmental variance & 36.47 & 18.06 & 49.53 & 20.1 \\
\hline Additive variance & $57.2 *$ & 48.03 & 58.3 & 88.4 \\
\hline Dominance variance & -17.8 & -16.6 & -49.1 & -42.7 \\
\hline$\chi^{2}(\mathrm{df})^{1}$ & $0.075(3) \mathrm{ns}^{2}$ & $2.89(3) \mathrm{ns}$ & $1.828(3) \mathrm{ns}$ & $15.47(3) \mathrm{s}^{3}$ \\
\hline Heritability $\left(\mathrm{h}^{2}{ }_{\mathrm{n}}\right)(\%)$ & 61 & 73 & 54 & 81 \\
\hline \multicolumn{5}{|l|}{$\begin{array}{l}\text { Number of heads per } \\
\text { plant }\end{array}$} \\
\hline Phenotypic variance & 28.4 & 5.24 & 11.43 & 9.38 \\
\hline Environmental variance & 6.78 & 4.76 & 9.29 & 3.962 \\
\hline Additive variance & 21.6 & 0.48 & 2.137 & 5.42 \\
\hline Dominance variance & -12.6 & -0.494 & -3.91 & -2.437 \\
\hline$\chi^{2}(\mathrm{df})$ & $0.296(3) \mathrm{ns}$ & $0.0684(3) \mathrm{ns}$ & $1.518(3) \mathrm{ns}$ & $1.344(3) \mathrm{ns}$ \\
\hline Heritability $\left(\mathrm{h}^{2}\right)(\%)$ & 76 & 9 & 19 & 58 \\
\hline \multicolumn{5}{|l|}{$\begin{array}{l}\text { Number of grains per } \\
\text { spike of the longest culm }\end{array}$} \\
\hline Phenotypic variance & 258.8 & 118.73 & 288.5 & 165.05 \\
\hline Environmental variance & 79.43 & 81.65 & 90.5 & 0.252 \\
\hline Additive variance & $179.4 *$ & 13.22 & $198.1^{*}$ & 164.8 \\
\hline Dominance variance & -65.6 & $23.88^{*}$ & -96.7 & -78.9 \\
\hline$\chi^{2}(\mathrm{df})$ & 0.147 (3) ns & $0.0058(3) \mathrm{ns}$ & $2.703(3) \mathrm{ns}$ & 1.9395 (3) ns \\
\hline Heritability $\left(\mathrm{h}_{\mathrm{n}}^{2}\right)(\%)$ & 69 & 11 & 69 & 99.8 \\
\hline \multicolumn{5}{|l|}{$\begin{array}{l}\text { Grain weight per spike of } \\
\text { the longest culm }(\mathrm{g})\end{array}$} \\
\hline Phenotypic variance & 0.843 & 0.2465 & 1.11 & 0.642 \\
\hline Environmental variance & 0.232 & 0.1843 & 0.564 & 0.172 \\
\hline Additive variance & 0.611 & 0.056 & $0.55^{*}$ & $0.47 *$ \\
\hline Dominance variance & -0.264 & 0.006 & -0.372 & -0.218 \\
\hline$\chi^{2}(\mathrm{df})$ & $2.534(3) \mathrm{ns}$ & $0.117(3) \mathrm{ns}$ & $0.0274(3) \mathrm{ns}$ & $1.096(3) \mathrm{ns}$ \\
\hline Heritability $\left(\mathrm{h}_{\mathrm{n}}^{2}\right)(\%)$ & 72 & 23 & 49 & 73 \\
\hline \multicolumn{5}{|l|}{ Grain yield per plant (g) } \\
\hline Phenotypic variance & 179.7 & 28.7 & 103.28 & 45 \\
\hline Environmental variance & 39.36 & 22.92 & 78.67 & 20.12 \\
\hline Additive variance & $140.3 *$ & 5.79 & 24.61 & $24.88^{*}$ \\
\hline Dominance variance & $-75.3^{*}$ & -5.72 & -54.48 & $-13.19^{*}$ \\
\hline$\chi^{2}(\mathrm{df})$ & $0.1689(3) \mathrm{ns}$ & $0.569(3) \mathrm{ns}$ & $0.127(3) \mathrm{ns}$ & $0.01(3) \mathrm{ns}$ \\
\hline Heritability $\left(\mathrm{h}_{\mathrm{n}}{ }_{\mathrm{n}}\right)(\%)$ & 78 & 21 & 24 & 55 \\
\hline \multicolumn{5}{|l|}{ Single grain weight (mg) } \\
\hline Phenotypic variance & 55.25 & 18.71 & 54.85 & 39.15 \\
\hline Environmental variance & 15.66 & 9.11 & 21.45 & 16.66 \\
\hline Additive variance & $39.59^{*}$ & 9.3 & $33.4^{*}$ & 22.49 \\
\hline Dominance variance & -20.1 & 0.3 & $-16.6^{*}$ & -4.492 \\
\hline$\chi^{2}(\mathrm{df})$ & $2.934(3) \mathrm{ns}$ & $1.863(3) \mathrm{ns}$ & $0.0125(3) \mathrm{ns}$ & $0.1398(3) \mathrm{ns}$ \\
\hline Heritability $\left(\mathrm{h}_{\mathrm{n}}^{2}\right)(\%)$ & 72 & 49.7 & 61 & 57 \\
\hline
\end{tabular}

${ }^{1} \mathrm{df}=$ degrees of freedom, calculated as the number of generations minus the number of estimated variance parameters.

${ }^{2} \mathrm{~ns}=$ non-significant $\chi^{2}$ test.

${ }^{3} \mathrm{~s}=$ significant $\chi^{2}$ test.

$*=$ statistically significant at $\mathrm{p}=0.95$.

The additive and dominance variance components estimates were inconsistent between crosses and across the testing sites. The values of narrow-sense heritability $\left(\mathrm{h}^{2}{ }_{\mathrm{n}}\right)$ varied depending on the testing site, crossing combination and trait analyzed. For the Soissons/Sana cross higher $\mathrm{h}^{2}{ }_{\mathrm{n}}$ values were observed for all traits (except for single grain weight) at the Nova Gradiska site, while for the Soissons/ Zitarka cross higher values were found for all traits (except for plant height) at the Osijek site. The $\mathrm{h}^{2}{ }_{\mathrm{n}}$ values for the Soissons/Zitarka cross were highest for plant height, single grain weight and grain yield per plant, whilst for the Soissons/Sana cross the $\mathrm{h}^{2}$ values were highest for the 
number of grains per spike of the longest culm, plant height and grain weight per spike of the longest culm.

\section{Discussion}

Although varying depending on the cross, experimental site and trait, in most cases the variation in the generation means fitted a digenic epistatic model which indicated that improvement of the traits studied would be moderately difficulty as compared to the situation pertaining had an additive-dominance model (best from a breeders point of view) provided the best fit. These findings correspond with the results published by some other authors (Pawar et al., 1988; Singh and Singh, 1992; Misra et al., 1994; Singh et al., 1998) but not with those reported by Sawant and Jain (1985). This situation is even more complicated when dominance effects are more important than additive effects, as was the case in this experiment.

For grain yield per plant in the Soissons/Zitarka cross at the Osijek site and single grain weight in the Soissons/ Sana cross at the Nova Gradiska site none of the models explained variation between generations, indicating more complex mechanisms of genetic control. Such a situation is the least favorable from a breeders point of view, suggesting that a revised selection strategy is needed for the early generations due to complexity of the gene effects occurring in these generations. These results are in accordance with reports published by other authors (e.g. Chatrtah et al., 1986; Srivastava et al., 1992; Amawate and Behl, 1995, Kathiria and Sharma, 1996).

To identify whether a cause of the model failure is the presence of higher order interactions or linkage effects there further analyses needs to be carried out with enough generations to fit a full trigenic interaction and linkage model. In respect of epistatic effects, additive $\mathrm{x}$ additive effects were more important than dominant $\mathrm{x}$ dominant effects and only complementary epistasis was observed.

This situation is more favorable than the presence of dominant $\mathrm{x}$ dominant effects and/or duplicate epistasis due to a greater chance of breeding success, and partially explains why heterosis was not as frequent as might be expected from the fact that dominance effects were more important than additive effects. Snape (1987) pointed out that a very common situation, especially when analyzing yield and yield components, is to find that the additive effect is small and non-significant while the dominance estimate is large and highly significant. Estimates of additive effects can be small due to a high degree of dispersion of increasing alleles between parents, and dominance can be small due to its bi-directional nature. This might explain why additive genetic component of variance $\left(\mathrm{V}_{\mathrm{A}}{ }^{*}\right)$ varied greatly, although a clear-cut relationship between additive effects and additive genetic variance could not be detected. On the other hand, the negative and non-significant estimates of dominance variance $\left(\mathrm{V}_{\mathrm{D}}{ }^{*}\right)$ seen in our study could have been due to sampling errors and/or the fact that basic generations are inefficient when used for determining dominance variance.

The estimated values of narrow-sense heritability $\left(\mathrm{h}_{\mathrm{n}}^{2}\right)$ varied for plant height $(54-81 \%)$, number of heads per plant (9-76\%), number of grains per spike of the longest culm (11-99.8\%), grain weight per spike of the longest culm $(23-73 \%)$, grain yield per plant $(21-78 \%)$ and single grain weight (49.7-72\%) depending on the crossing combination and testing site. These heritability values are in accordance with those reported by other authors (Sidwell, 1978; Baric, 1996 and Drezner, 1996).

From a breeders point of view, the heritability estimates from both sites show that the Soissons/Zitarka cross has the greatest chance of genetic improvement in the number of heads per plant, grain yield per plant and single grain weight, while the Soissons/Sana cross has the greatest chance of increase in the number of grains per spike and grain weight per spike of the longest culm. The higher $\mathrm{h}^{2}$ values found at the Osijek site can be partially explained by the fact that the experimental field layout was rectangular at the Osijek site and square at the Nova Gradiska site. The estimates of heritability suggest that it would be better to breed the Soissons/Zitarka cross at the Osijek site and the Soissons/Sana cross at the Nova Gradiska site, suggesting that success in a breeding program could be under the influence of environment, even as regards specific genetic effects. These results show that both the adequacy of certain modes of inheritance as well as the importance and significance of gene effects for a certain trait were dependent upon the particular cross and experimental site, stressing the importance of the appropriate selection of both parents and breeding site for the success of a program.

\section{References}

Amawate JS and Behl PN (1995) Genetical analysis of some quantitative components of yield in bread wheat. Indian Journal of Genetics 55:120-125.

Baric M (1996) Kombinacijska sposobnost i nasljednost nekih kvantitativnih svojstava psenice (Tr. aestivum L.). $\mathrm{PhD}$ thesis, University of Zagreb, Zagreb.

Bartlett MS (1937) Some examples of statistical methods of research in agriculture and applied biology. J R Statist Soc Suppl 4:137-183.

Chatrtah R, Satija DR and Gupta VP (1986) Genetic analysis of grain yield in wheat. Indian Journal of Genetics 46:466-471.

Drezner G (1996) Selekcija psenice (Tr.aestivum L.) u ranim generacijama zavisno o kriterijima izbora. $\mathrm{PhD}$ thesis, University of Zagreb, Osijek.

Hayman BI (1960) Maximum likelihood estimation of genetic components of variation. Biometrics 16:369-381.

Kathiria KB and Sharma RK (1996) Genetic analysis for earliness, plant height and kernel weight in two breadwheat crosses under normal and saline sodic soils. Indian Journal of Genetics 56:548-552.

Kearsey MJ and Pooni HS (1996) The genetical analysis of quantitative traits. 1st edition. Chapman \& Hall, London, 381 pp. 
Lynch M and Walsh B (1998) Genetics and analysis of quantitative traits. Sinauer Associates, Inc., Sunderland, 980 pp.

Mather K and Jinks JL (1982) Biometrical genetics. 3rd edition. Chapman \& Hall, London, 396 pp.

Misra SC, Rao VS, Dixit RN, Surve VD and Patil VP (1994) Genetic control of yield and its components in breadwheat. Indian Journal of Genetics 54:77-82.

Pawar IS, Paroda RS and Singh S (1988) Gene effects for six metric traits in four spring wheat crosses. Indian Journal of Genetics 48:195-199.

SAS STAT Software 6.12. Release (1989-1996) Copyright (c) by SAS Institute Inc., Cary, NC, USA.

Sawant AR and Jain KB (1985) Gene action for certain quantitative characters in common wheat in optimal and suboptimal environments. Indian Journal of Genetics 45:376-384.

Sidwell RJ, Smith EL and McNew RW (1978) Heritability and genetic advance of selected agronomic traits in a winter cross. Cereal Research Communications 6(2):103-111.
Singh G, Nanda GS and Sohu VS (1998) Gene effects for grains per spike, grain weight and grains per spikelet in a set of nineteen crosses of wheat. Indian Journal of Genetics 58:83-89.

Singh RP and Singh S (1992) Estimation of genetic parameters through generation mean analysis in breadwheat. Indian Journal of Genetics 52:369-375.

Snape JW (1987) Conventional methods of genetic analysis in wheat. In: Lupton FGH (ed) Wheat breeding, its scientific basis. Chapman \& Hall, London, New York, pp 109-128.

Srivastava RB, Sharma SC and Yunus M (1992) Additive and nonadditive gene effects for yield and yield components in two wheat crosses. Indian Journal of Genetics 52:297-301.

Whalia DP, Tashi Dawa and Plaha P (1994) Genetics of yield components in spring wheat. Cereal Research Communication 22:185-186.

Editor: Marcio de Castro da Silva Filho 